\title{
Virtudes públicas en las inscripciones funerarias latinas: la expresión de la fortaleza y elocuencia femeninas
}

Public Virtues in Latin Funerary Inscriptions as the Expression of Women's Strength and Eloquence

\author{
Ma. Teresa MuÑoz GARCÍA DE ITURROSPE \\ Universidad del País Vasco / Euskal Herriko Unibertsitatea \\ maite.munoz@ehu.es
}

\begin{abstract}
Resumen: Los epitafios latinos dedicados a mujeres de toda condición encomian con frecuencia las virtudes de la puella y de la matrona ejemplar, eminentemente domésticas. Sin embargo, valores considerados masculinos como la fortaleza, la justicia y la elocuencia son ocasionalmente subrayados en inscripciones tanto métricas como en prosa, con una finalidad fundamentalmente consoladora que, sobre todo en las inscripciones cristianas, sirve además para completar un nuevo modelo de comportamiento.
\end{abstract}

ABSTRACT: Latin epitaphs dedicated to women of all condition often praise the virtues, mainly domestic, of some exemplary puellae and matronae. However, masculine values considered like strength, sense of justice, or eloquence are occasionally underlined in carmina epigraphica as well as in prose inscriptions, with a mainly consoling aim which, above all in Christian inscriptions, is used to complete a new model of behavior.

PALABRAS ClAVE: Epigrafía latina; mujeres; epitafios; retórica; elogio.

KeYwORDs: Latin Epigraphy; Women; Epitaphs; Rhetoric; Praise.

RECIBIDO: 31 de diciembre de 2014 - ACEPTADO: 2 de marzo de 2016.

DOI: $10.19130 /$ iifl.nt.2016.33.2.709

...they remain, something that has been stated and must eternally endure.

Virginia Woolf, "On Not Knowing Greek"

Provistas aún en nuestra percepción de una cierta rigidez y solidez estática y no menos valerosas y fuertes que los grandes personajes femeninos de la tragedia griega, cuyas palabras, como Woolf ensalzaba en su ensayo Sobre no saber Griego (1986, p. 28) "permanecen, son algo que se ha pronunciado y debe perdurar eternamente", por sus palabras y por sus casi sin excepción limitados actos, las mujeres romanas, desde la República a la Antigüedad tardía, evidencian esa continuidad en algunos de los elogios que se grabaron sobre sus tumbas. 
Las inscripciones antiguas, a las que no pocas mujeres a menudo privilegiadas asoman, muestran de vez en cuando esa consabida faceta familiar y doméstica que esconde una fortaleza y una relevancia genuinas. Los elogios de la esposa, de la hermana o de la hija y las loas de la concordia conyugal o familiar refuerzan la autoridad moral en la sociedad de su cónyuge o del padre, de la misma manera que el elogio de la niña muerta antes de tiempo sirve a menudo para sublimar la consideración más positiva de su familia. Es por esta finalidad que Ausonio inicia el poema dedicado a su consuegra, la noble Pomponia Úrbica, ligando su linaje y las viejas tradiciones que lleva consigo al enriquecimiento enseñado por su esposo, pero también por su padre y su madre:

ingenitis pollens virtutibus auctaque et illis

quas docuit coniunx, quas pater et genetrix (Aus., Parent., 30.3-4).

La pervivencia de la memoria es muy clara en monumentales encomios en piedra, ya de por sí escaparates, como el encargado por un hombre tan influyente y poderoso como Vetio Agorio Pretextato para reconocer las virtudes domésticas pero sobre todo cívicas de su esposa Aconia Fabia Paulina (CIL VI, 1779), pero no parece menos importante para esos otros esposos que lloran contando y haciendo grabar los años (y muy a menudo meses y años) de concordia coniugalis.

Aquí vamos a destacar las inscripciones funerarias, muchas de ellas métricas, compuestas con una intención que va más allá de la mera constatación del elogio estereotipado, género poco abierto a la innovación y tendente a reconocer de manera indirecta la valía de las mujeres. La escena de la dulce y pura Nausícaa ante Odiseo al final del canto sexto representa, con brevedad y a la vez encanto, este valor: ella le pide que una vez en el palacio se dirija a saludar primero no al rey Alcínoo, "como un inmortal" en su trono, sino a la reina Arete (es decir, 'virtud, excelencia'), que está bordando con sus esclavas, merecedora de que el viajero le abrace las rodillas para así ganar un bienestar como el de su propia casa, puesto que por merece siquiera la pena detenerse ante el rey; la madre tiene que ser su primer objetivo (Od., 6.303-315). Esa certeza práctica es en Roma pronto corroborada por Catón el Censor, quien en uno de sus preceptos o apotegmas - tomados de Temístocles y evocados por Plutarco - sanciona un poder universal, que parte de lo que pasa en el interior de todas las casas de los pujantes romanos: 


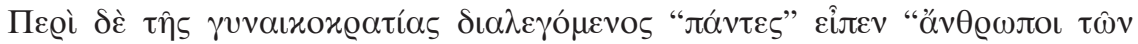

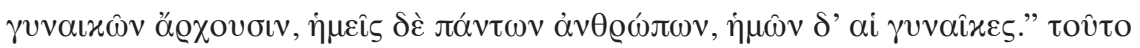

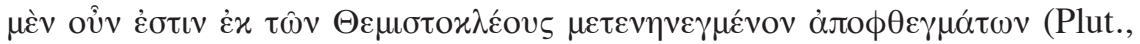
Cat. Ma., 8, 4). ${ }^{1}$

La representación en el elogio epigráfico de este dominio y de su alcance contrasta con el retrato que aporta la literatura misógina, poblada de mulierculae, vilis plebecula, inperitae (así, Varr., L. L., 5.73, mulier ... a mollitie), ${ }^{2}$ ignorantes, tontas a las que se recomienda callar, como aún se presentan La dama boba de Lope de Vega o Las preciosas ridículas y Las mujeres sabias de Molière.

En la epigrafía funeraria latina, además de las más estereotipadas menciones de las labores propias de la matrona, vidua o la virgo intachables, surgen de forma esporádica alusiones a una influencia en la esfera pública, evidente en intervenciones tan notorias como la enunciación del mismo discurso funerario. ${ }^{3}$ Estas virgines y matronae consiguen así distinguirse del resto en gran medida por su fuerza y resistencia, reconocidas por alguna capacidad intelectual o espiritual que explica su salto a una dimensión exterior, convirtiéndose en nuevos modelos de comportamiento para nuevas generaciones de madres e hijas. De hecho, algunos elogios femeninos pretenden dar nueva vida a unos valores ya grabados en lugar preferente en uno de los ejemplos más señeros de la literatura arcaica romana, el elogio en saturnios de Lucio Cornelio Escipión Barbado:

Cornelius Lucius Scipio Barbatus Gnaivod patre prognatus, fortis vir sapiens quoius forma virtutei parisuma / fuit ...

$(C I L \mathrm{VI}, 1284=C L E 7)$

Este recorrido parte de una inscripción métrica que una orgullosa Plancina refiere en primera persona, anteponiendo su origen ilustre y la

1 "Hablando sobre el poder de las mujeres dijo: «Todos los hombres mandan sobre sus mujeres; nosotros, en cambio, sobre todos los hombres, pero somos mandados por nuestras mujeres». Esto está tomado de las sentencias de Temístocles...” (trad. de J. M. Guzmán Hermida y Ó. Martínez García).

${ }^{2}$ Aún Pedro de Cella, Comm. in Ruth, 2: "Virtutes enim virum faciunt, vitia mollitiem femineam semper pariunt".

${ }^{3}$ Prescendi 2008, esp. pp. 301-305. 
maternidad ejemplar a un matrimonio igualmente modélico, pero algo relegado. ${ }^{4}$ Buena madre y buena esposa (en lugar del superlativo opti$m a$ o similar), ${ }^{5}$ hasta tal punto que, ayudada por su condición reiterada de noble (seguramente de una estirpe indígena) y de matrona honesta, consiguió superar a las demás mujeres (prima mulierum, praeter alias feminas) en una especie de competición, habitual en las inscripciones honoríficas de las más altas magistraturas: ${ }^{6}$

Numidarum prima mulierum
Plancina genere regio
bona mater bona coniunx
hic sum sepulta multis
[1]acrimis meorum amaris
matrona honesta praeter alias femina[s]
hic sum sepulta exorta genere regio
ter denos annos et ter ternos functa cur[a]
bonarum feminarum Q(uintus) Arruntius Mas[cel(?)]

$(C I L \text { VIII, } 16159=\text { CLE 1554, Le Kef })^{7}$

El redundante nacimiento privilegiado se sitúa en tres lugares destacados: prima mulierum (¡recordando a sus lectores el comienzo del poema de Lucrecio, con su dedicatoria a Venus, Aeneadum genetrix!), genere

${ }^{4}$ Gascou 1969, p. 567, defiende que esta Plancina puede identificarse con la Maria Plancina mencionada en la inscripción en prosa de otra matrona "de ilustre memoria" de la cercana Djezza; asimismo, en el África Proconsular: "D(is) M(anibus) s(acrum) / Sertoria / Saturni/na Mari/ae Planci/nae filia / Licini For/tunati / coniux ca/rissima / h(onestae) m(emoriae) m(atrona) / vixit an/nis LXXV / h(ic) s(ita) e(st)" (ILTun 1633).

${ }^{5}$ A veces es más eficaz el lacónico y central superlativo, entre el nombre propio y la categoría jurídica de esposa: "Avita optima uxor" (ICUR VIII, 20921, cementerio de Santa Inés).

${ }^{6}$ Así, CIL II 4105 (Tarraco): “Devictori omnium gen/tium barbararum et su/per omnes retro princi/pes providentissimo / Imp(eratori) Caes(ari) [[[Val(erio) Lici]]]/[[[niano Lici]nio]]...".

7 "La primera de las mujeres númidas, Plancina, de estirpe real, buena madre, buena esposa, aquí estoy enterrada con las muchas lágrimas amargas de los míos, una mujer honrada más que las demás; aquí estoy enterrada, nacida de familia real, tras cumplir durante treinta y nueve años con las obligaciones de las mujeres nobles. Quinto Arruncio Mas [ ]". Ésta y las demás traducciones de carmina Latina epigraphica son de C. Fernández Martínez 1988. 
regio, exorta genere regio. ${ }^{8}$ De la misma manera, su condición de “cumplidora de las funciones propias de las buenas mujeres" viene señalada al final, cerrando perfectamente el elogio inicial: bona mater, bona coniunx, cur[a?] bonarum feminarum. ${ }^{9}$ Con una complejidad métrica destacable - los versos 1, 2, 4 y 5 son dímetros yámbicos, 6 y 7 trímetros yámbicos, 3 y 8 jónicos menores - , la firma queda relegada al final, con el escueto nombre del dedicante (o el marido o un hijo), probablemente un ciudadano romano de origen númida.

En la misma provincia hay otro noble local (su nombre completo, en dos versos, Marcus [...] / Aemilius Primus Flavianus) que comparte el epitafio métrico dedicado por dos hijos con su esposa Julia (concordes animae... cum vita maneret, en el primer verso), dedicándosele a ella siete versos, que ensalzan que fue uidua y antes de ello coniux y siempre modelo para las demás mujeres (femineis exemplar moribus omne): ${ }^{10}$

... dulci viduata marito

coniux / ${ }^{15}$ casta suum per se solata labo/rem,

Iulia femineis exem/plar moribus omne

Seti/na excelso genere orta et glo/ria gentis,

hic sita, sed sedes $/ 20$ meruit penetrare piorum;

Elysios celebrat caro coniuncta marito,

Sexsies hu / ic decimum spatium compleve / rat annus.

$(\text { CIL IX, } 2340=\text { CLE 1969, vv. 9-15, Madaura })^{11}$

${ }^{8}$ Res Gestae Divi Augusti, 27: "ex regio genere Armeniorum oriundus"; Aún en el siglo VII, "Hic quiesc[e]t in pa//ce b(onae) m(emoriae) [M] aria, claro / veniens de stirpe pa/rentum, cui $\mathrm{t}$ [ri]bus ann(is) / trib(us) tantu $<\mathrm{m}>\mathrm{li}[\mathrm{c}]$ uit / dulci esse coniun//cta marito, quem / invida mors XVII / etatis anno trans/acto dulce priva/vit iugale” (CI IX, 58, Albingaunum).

${ }^{9}$ Más sencilla, en una línea central, matrona, un homenaje semejante, sólo a la esposa, quizá ya cristiana: "C. Chiriache / matrona / co(n)iux bona” (CIL VI, $13671=$ ILCV 0378i-j, Roma).

${ }^{10} \mathrm{Cmp}$. CIL VI, 1706 (de un vir clarissimus): “...ob egregia facta et ra/rum veteris sanctitatis / exemplar inlustrat $<0=\mathrm{V}>$ ri / prosapiae suae cuius ope / auctam instauratamq(ue) / tota se Piceni et Flami/niae provincia gratu/latur ..."; CIL VI, 1741: "genere nobili domi forisque" (para Memmio Vitrasio Orfito, prefecto urbano con el emperador Constancio II).

11 “...su virtuosa esposa, Julia Setina, privada de tan buen marido, reconfortando a solas su pesar, todo un ejemplo de conducta femenina, nacida de ilustre linaje y gloria de su familia, aquí está enterrada, pero ha merecido entrar en la morada de los santos. Unida a su querido esposo, recorre el Elíseo. Dieciséis años le había dedicado...”. 
De estructura muy semejante a la del epitafio de Plancina, con una disposición en la que prima el origen noble de la familia, luego la maternidad y solamente en el tercer miembro el matrimonio, otra inscripción del norte de África termina con la mención expresa de la necesidad de pervivencia de la memoria para un monumento con los nombres y edades de sus cinco hijos (todos identificados con el gentilicio abreviado, $P$ ) aquí en la voz consciente del marido vivo: memoriam feci perennem. ${ }^{12}$ Se trata de recuperar a través del epígrafe a una familia perdida, encabezada de nuevo por la expresión honesta femina,${ }^{13}$ condición asimismo reforzada por la torpe expresión que le sigue, bonis natalibus nata:

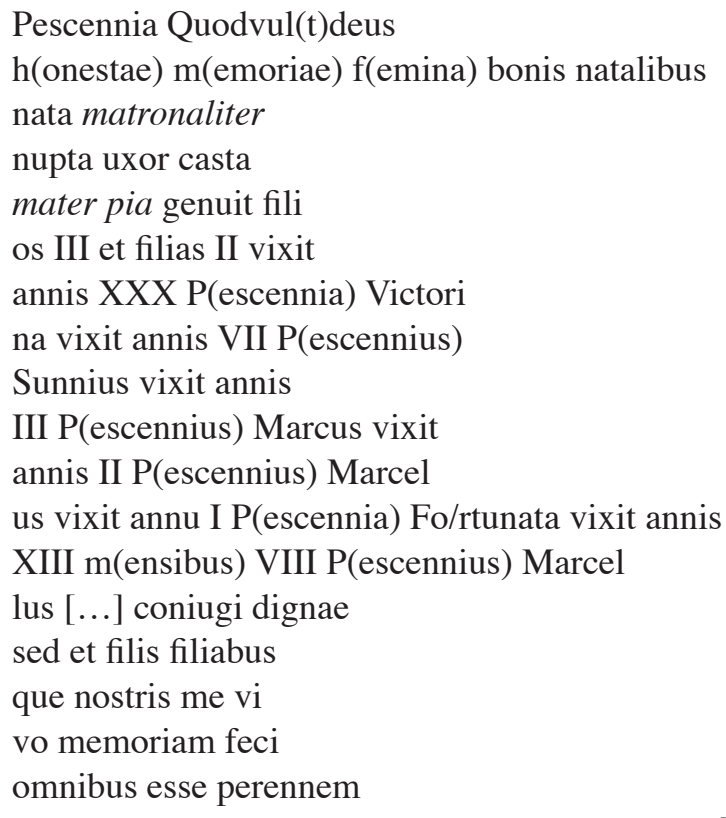

(CIL VIII, 870, Giufi)

12 Esposo e hijos también dedican en Vienne un monumento eterno, en el que se duplican las funciones ejemplares, de manera que maternidad y matrimonio merecen cuatro superlativos: "memoriae (a)eternae / Iul(iae) Severin(a)e matronae honestis/ sim(a)e coniugi karissim(a)e matri pi/entis(s)im(a)e parenti dulcis(s)im(a)e" (CIL XII, 1972).

${ }^{13}$ Benzina-Ben Abdallah y Ladjimi-Sebaï 1977, p. 164, reúnen once inscripciones de mujeres honestae en la provincia y concluyen que este calificativo designa a esposas de soldados, miembros de la nobleza municipal y a tres flamínicas. 
El adverbio matronaliter ${ }^{14}$ - desconocido para las fuentes jurídicas aunque confirme un iustum matrimonium - sólo se halla en esta inscripción funeraria y en la primera parte de uno de los más antiguos textos cristianos escritos en latín, la Passio Perpetuae et Felicitatis, dedicada a la mártir asimismo de nacimiento noble y africana Perpetua. En el caso de la mártir se indica - a partir de lo que ella misma refiere que escribió y de hecho se narra en primera persona (2, 3: "haec ordinem totum martyrii sui iam hinc ipsa narrauit sicut conscriptum manu sua et suo sensu reliquit") - que cuenta con el sostén cara a la memoria futura de sus actos dadas su procedencia de una buena familia y una buena educación "liberal, abierta", completadas por su realización como madre:

Vibia Perpetua, honeste nata, liberaliter instituta, matronaliter nupta...

(ibid., 2, 1)

Advertimos que el autor de la versión griega de la Passio encontró una dificultad a la hora de entender matronaliter nupta, debido al carácter prácticamente de hápax del adverbio, por lo que modificó la expresión, utilizando un adverbio más ordinario, ‘̇̌ó $\chi \omega \varsigma$ ('de forma conveniente'):

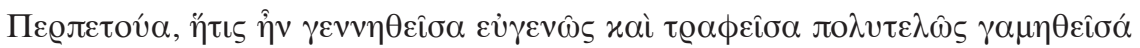
$\tau \varepsilon \dot{\varepsilon} \xi o ́ \chi \omega \varsigma$.

Perpetua sería, también a partir de los epítetos que la caracterizan los difusores de su retrato literario, fortissima y nobilissima, representación semejante a la que se diseña para los emperadores en sus inscripciones honoríficas. ${ }^{15}$ Cercana a la virago o a la mulier virilis, se señala la particularidad de su maternidad, que es la que le otorga una condición de matrona a la que finalmente acabará renunciando incluso con un gesto

${ }^{14}$ En una línea central, matrona, un homenaje semejante, sólo a la esposa (CIL VI, 13671, Roma): “C. Chiriache / matrona / co(n)iux bona”; también como sinónimo de mater, aquí destacando en el epitafio la mención de "madre de...", "Nunita qu(a)e fuit matrona diaconi\{s\}" (CIL VI, $41342=$ ICUR V, 13355). Asimismo, más lacónico, aún con superlativo para elogiar a la buena esposa: "Terentia ma/trona con/iux castissimi / in pace" (ICUR IV, 12770).

${ }^{15}$ Así, CIL VI, 1125 (inscripción honorífica de Maximiano, ca. 285): "Magno et invicto ac super omnes retro principes fortissimo, Imp(eratori) Caes(ari) M(arco) Aur(elio) Valerio / Maximiano ...". 
violento (proiciens infantem, 6.6), siquiera sólo en la versión de los Acta Perpetuae. ${ }^{16}$

En el caso de Pescenia por la relevancia de los hijos y en el de la mártir Perpetua por la ausencia del esposo en el relato, bien parece que pudiera entenderse la expresión matronaliter nupta como "casada para ser madre". De otro lado, consideramos que el autor de la inscripción, dada su procedencia, pudo conocer la pasión de su mártir, compuesta en los primeros años del siglo III y cuya lectura acabó siendo recomendada por Concilios del siglo IV , como los de Hipona de 393 y Cartago de $397 .{ }^{17} \mathrm{El}$ igualmente africano Tertuliano emplea en una mención de encomio de la misma mártir Perpetua la expresión fortissima martyr (Tert., An., 55.4), ${ }^{18}$ que pasa esporádicamente para describir con brevedad a otras mártires ${ }^{19}$ y que se graba para una joven cristiana de Roma:

\author{
Elia Valeria fortissima ${ }^{20}$ que vix(it) annis XVIII \\ $\mathrm{m}$ (ensibus) XI die(bu)s XXVIII in pace \\ (ICUR X, 26989, cementerio de Basila, en la vía Salaria)
}

Con el sentido de audax, animosus, audax, non timidus propio de la comedia latina (como en Ter., Andr., 445), en las inscripciones tenemos una joven virgen cristiana fortissima de Roma, que no es otra que la

16 Weitbrecht 2012, pp. 153-155, advierte la importancia en la Passio de la maternidad de Perpetua y del rechazo por la causa en los Acta. Por su parte, Cobb 2008, pp. 176 ss., defiende la tensión entre rasgos masculinos y femeninos, que el narrador intenta destacar en estas primeras frases del relato martirial.

${ }^{17}$ Entre 400 y 420, San Agustín pronuncia tres sermones (nos. 280-282), en los que refiere los maravillosos efectos de la lectura pública de este relato, que por tanto también pudo conocer el autor del epitafio de Pescenia.

${ }^{18}$ Sirve para encomiar el arrojo y mérito militar por ejemplo del emperador Constancio II en un pedestal honorífico, datado entre 353-360 d. C., que se le dedica en Córdoba: "Fortissimo indulgentissimo / principi domino nostro / Constantio victori / perpetuo semper Augusto / Decimius Germanianus / vir clarissimus consularis / provinciae Baeticae / numini maiestatique / eius dicatissimus" (CIL II2/7, 265; CIL II 2206; CIL X, 748, 2).

${ }^{19}$ Así, la mártir Donata es fortissima femina (Aug., sermo 299e, in natale martyrum Scilitanorum in basilica Novarum).

${ }^{20}$ Los editores optan por entender Fortísima como un cognomen. Consideramos, sin embargo, la lectura de un epíteto superlativo, que denota una cierta antigüedad. Así entendido, el titulus destacaría asimismo por los duo nomina de la joven difunta; el segundo gentilicio venía a ser herencia de la madre o de otro pariente, y su expresión junto al otro nomen podría indicar una pertenencia a una cierta clase superior. 
santa mártir Faustina, ${ }^{21}$ quien merece un lacónico epitafio sólo roto por el superlativo:

Faustinae virgini fortissimae

que bixit ann(os) XXI

in pace

(ICUR III, 8879, catacumbas de Santa Ciriaca, en la vía Ostiense)

También ya para finales del siglo IV este adjetivo, aún en el grado superlativo característico de los elogios más antiguos, se lee para una cristiana romana ya claramente como nombre:

dep(osita) in p(ace) XV kal(endas) sep(tembres)

Fortissima cons(ulibus) Timasio et Promoto v(iro) c(larissimo)

(ICUR VII, 17497, año 389, cementerio de Ciriaca)

Otro gran elogio fúnebre en piedra, el dedicado en la segunda mitad del s. II por su patrono a Alia Potestad, apodada por él cariñosamente Perusina, coloca el calificativo fortis en el primer lugar del elenco que empieza en el verso 8 e insiste en la importancia del elogio para la pervivencia de una mujer fuerte, capaz de hacer de dos amantes unos Pílades y Orestes que sólo aguantan por su mediación: ${ }^{22}$

fortis sancta tenax insons fidissima custos munda domi sat munda foras notissima volgo.

$(C I L$ VI, $37964=$ CLE 1988, vv. 8-9)

A continuación la morosa y sensual descripción física, que acaba con las piernas de Alia, conduce un tanto abruptamente en su comparación con Atalanta ("quid crura? Atalantes status illi", v. 21). Belleza, ejemplaridad (y santidad) a menudo van unidas en todas las culturas, y de hecho el término fortis se adecua y alimenta perfectamente esta ambigüedad,

${ }^{21}$ Hay un mártir, asimismo, "muy valeroso", San Primitivo, en el cementerio de Ostriano: "Primitius in pace qui post / multas angustias / fortissimus martyr / et vixit annis p(lus) m(inus) / XXXVIII coniugi suo / perdulcissimo bene merenti / fecit" (ILCV 01993a).

22 "Quantumq(ue) tamen praeconia nostra valebunt, / versiculis vives quandiucumque meis" (vv. 17-18), es decir, "cuanto mejor sea mi elogio, tanto más tiempo permanecerás viva en mis pobres versos". 
siendo un calificativo que en Plauto se emplea con un sentido más cercano al de formosus o pulcher (así, Bacch., 216-217; Mil., 1106). Es otro comediógrafo, Afranio, quien, desde la fabula togata, presenta con ironía la hermosura como la mitad de una dote en sí misma para una virgo casadera, siendo la otra parte de "extra" la fortaleza, ya independiente:

Formosa virgo est: dotis dimidium vocant

isti, qui dotis neglegunt uxorias:

praeterea fortis.

(Afran., fragm. 156-158, p. 158)

La excepcionalidad subyace en cuestiones retóricas como la bíblica que se lee en el libro de los Proverbios, "Mulierem fortem quis inveniet?" (Prov. 31.10), ${ }^{23}$ de tanta repercusión, máxime cuando Bernardo de Claraval (Ep. 113.1) la interpreta como figura de la Virgen y sirve a Fray Luis de León para dar comienzo a La perfecta casada y a Calderón para titular ¿Quién hallará mujer fuerte?, uno de sus autos sacramentales. En este sentido, no podemos descuidar que en otro versículo de otro libro bíblico de sentencias los epítetos son bona y fortis, cualidades cuyos efectos revierten directamente sólo en el esposo: "Mulieris bonae beatus vir: numerus enim annorum illius duplex; mulier fortis oblectat virum suum $^{24}$ et annos vitae illius in pace implebit" (Eccli. 26, 1-2). Es el mismo modelo que, en el apócrifo libro de Tobías, sirve para describir a Sara $(6,12)$ : "haec puella sapiens et fortis et bona valde et constabilita". 25

Por otro lado, como ocurre con el de la propia Perpetua, a veces de forma voluntaria los mismos nombres de las difuntas sirven para apuntalar el elogio ejemplar de la fortaleza y el valor, a menudo vinculados de nuevo con el origen noble y la maternidad, cualidades que favorecen la recta pervivencia de la estirpe.

Es el caso de la bella y noble Sólida, con un cognomen muy raro, que abre explícitamente el siguiente carmen epigraphicum cristiano de

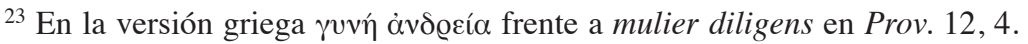

${ }^{24}$ Nótese la aclaración, con cursivas en el original, inserta en la traducción de mulier fortis y de esta parte del versículo por F. Torres Amat (Madrid, 1834): "La muger fuerte ó varonil es el consuelo de su marido, y le hace vivir en paz los años de su vida"; en la misma versión mulier bona se traduce como "muger virtuosa".

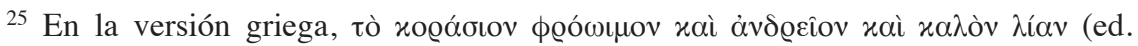
Wagner 2003, p. 68). 
Aquileya, en cuya parte central el esposo-autor se dedica a lamentar los hijos no tenidos y a expresar, con un apóstrofe dirigido a sí mismo, que pro meritis de ella dedica un hermoso y resistente monumento de mármol para ambos:

Nomine quae Solida vixit cum coniuge sanctae clara genus ${ }^{26}$ et pulcra gena ${ }^{27}$ sed plena pudores.

Hic iacet extremum fatis opressa sinistris, hec eadem nox una dedit talamosque necemque aurea bis denos aetas cum stringeret annos, quatuor ast dederat solacia digna marito; pignore caro tamen vacuum soluta cubile pergit ad occasus, quoniam sors omnia voluit cuius pro meritis pulcram de marmore sedem. Valeriane, dolens comuni in pace parasti / deposita IIII Idus Septembris $(C I L \mathrm{~V}, 1710=C L E 00640)^{28}$

Aunque no podamos tener siempre la certeza, al menos en estos casos parece que los autores de estos carmina piensan especialmente en los nombres de las difuntas para reafirmar su modelo ejemplar de castidad (como la Kasta Magni raris/sima coniunx, en Hamdoune 2011, ${ }^{\circ} 19$, Haïdra. s. III), esperanza (Helpis, cit. infra) y prudencia en el siguiente epitafio, de una univira y vidua, con su nombre después de los altos conceptos de iustitia, pietas y fides, y sin expresión propiamente cristiana alguna:

${ }^{26}$ Un poema funerario dedicado por un hermano a una virgo consagrada de Milán - clara genus censu pollens et mater egentum / virgo sacrata deo Manlia Daedalia, "noble, rica en hacienda y protectora de los necesitados" (CIL V, $6240=C L E 1434$, $3-4)-$, con la misma esperanza de que sus palabras "las lean una y otra vez los tiempos venideros" ("haec... quae relegant olim saecla futura", v. 10).

${ }^{27}$ Quizá eco bíblico, precisamente del Cantar de los Cantares (1, 9): "Pulchrae sunt genae tuae sicut turturis".

28 "De nombre Sólida, la que vivió sin mácula junto con su esposo, de linaje ilustre y de hermoso rostro, pero llena de pudor, yace aquí, totalmente destruida por su adverso destino. A ella una misma noche le proporcionó a la vez el tálamo nupcial y la muerte, cuando su dorada edad rozaba los veinte años. Y había sido además, durante cuatro, un digno consuelo para su marido. Pero, dejando su lecho desprovisto de algún hijo al que hubieran amado, se dirige hacia su muerte, pues así ha girado su destino. Por todos sus merecimientos y lleno de dolor, una hermosa morada de mármol, Valeriano, preparaste, para el descanso de ambos". 
Prae cunctis opibus laudem servavit honesti

iustitia pietate fide Prudentia sollers

sancta pudica decens uni devota marito

cuius ab occasu vincli pertesa iugalis

octo et bis senis vidua permansit in annis

natorum curam miro suspexit amore

vixit annis LXVII m(ensibus) V d(iebus) XVIIII dep(osita) VIIII Id(us)

[Octobris.

$(\text { ICUR II, } 04415=\text { CLE 736 })^{29}$

Incluso, el ser agradable con todos es un elogio que se expone mejor cuando el cognomen es grata, y a la que dedica el juego de los acrósticos para desvelar su nombre, que así aparece al principio absoluto, antes de empezar la parte métrica, y al final:

\section{Veturia Grata //}

Vel nunc morando resta qui perges iter

etiam dolentis casus adversos lege

Trebius Basileus coniunx quae scripsi dolens

ut scire possis infra scripta pectoris

rerum bonarum fuit haec ornata suis

innocua simplex quae numquam serbabit dolum

annos quae vixit XXI et mensibus VII

genuitque ex me tres natos quos reliquit parbulos

repleta quartum utero mense octavo obit

attonitus capita nunc versorum inspice

titulum merentis oro perlegas libens

Cagnosces nomen coniugis Gratae meae.

$$
(C I L \text { VI, } 28753=C L E 108)^{30}
$$

29 “Con todas sus fuerzas preservó la virtud de la honestidad, bien diestra en asuntos de justicia, religiosidad, fe, moderación; santa, virtuosa, decente y entregada a un único marido. Desde su muerte, aborreciendo el vínculo matrimonial, permaneció viuda durante veinte años. Prestó atención a sus hijos con un cariño digno de admiración".

30 "Quédate un momento y detén tu paso, tú que vas caminando, y lee la adversa fortuna de este que se lamenta, para que puedas conocer los versos que salen de mi corazón y que yo, su esposo Trebio Basileo, lleno de dolor, he grabado. Ella estuvo adornada de toda clase de bondades para los suyos, honrada, sencilla, sin dejarse tentar por el engaño; vivió veintiún años y siete meses y engendró conmigo tres hijos, a los que dejó pequeños, y murió con el cuarto en el vientre, en su octavo mes; lee ahora atentamente los comienzos de cada uno de los versos, y te ruego que leas, por favor, el epitafio de quien tanto lo merece: conocerás así el nombre de mi esposa Grata”. 
La imagen de la justicia, que según defiende Lactancio constituye el fundamento de la fides coniugalis, ${ }^{31}$ ocupa el primer lugar de otro largo carmen epigraphicum seguido de un epitafio en prosa, que incluye con morosidad los datos biométricos que un doliente esposo dedica a una joven difunta de poco más de diecisiete años, en un suntuoso sarcófago datado en el año 364. Así, en el lugar central una vez destacado el vínculo del matrimonio llega la alusión directa al renombre y reconocimiento abierto en Roma (publica fama) gracias a la concordia coniugalis y a la condición de Acilia de univira, elementos que refuerzan el potente inicio:

Iustitiae facies pudor integer omnis honestas

casta maritali semper devincta pudore

$[\ldots]$ / scit publica fama

et probat omne bonum soli servasse marito. ${ }^{32}$

Fl(avius) Crescens Aciliae Baebianae dolcissimae adque amantissimae coniugi bene merenti in pace quae vixit annos decem et septem / et mensibus novem diebus tredecim fecit cum marito annum et mens(es) III dep(osita) VIII / Kal(endas) Mart(ias) Valentiniano et Valente Augg(ustis) iter(um) conss(ulibus).

(ICUR X, $27296=$ CLE 652, cementerio de la basílica de San Valentín $)$

Aunque de manera indirecta, mención aparte merecen las univirae, mujeres valerosas en cuanto que voluntariamente no vuelven a casarse después de enviudar. ${ }^{33}$ Así, en el epitafio de una viuda, el hijo elogia a su madre porque se mantuvo fiel al esposo, haciendo con él de padre y madre ("officium nato patris matrisque gerebas", ICUR II $6018=$ CLE 2103, v. 5 , cementerio de Comodila), sin más amor, lo que es el único mérito que puede celebrarse de una mujer ("unica materia est quo sumit femina laudem / quod te coniugio exibuisse doces", ibid., vv. 10-11).

Una vez más en primera persona, otra difunta toma la voz y la pala-

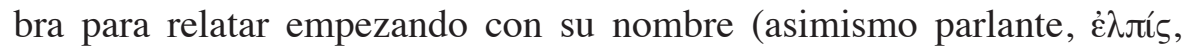

${ }^{31}$ Lact., Inst. div., 3.21.5: "Ubi est igitur virtus castitatis? ubi fides coniugalis? quae si tollas, omnis iustitia sublata est?” (cf. CIL VI, 38922: “... pr]oba fi[delis coni]ugalis / [de qu]a ego nihil / questus sum usque / in diem mortis nec / illa de me").

32 "Imagen de la justicia, pudor irreprochable, cúmulo de honestidad, virtuosa y ligada siempre a la lealtad conyugal [...] Los rumores de la gente saben qué hogar tan puro ha habido junto a ti y aprueban que hayas reservado toda clase de bienes sólo para tu esposo".

${ }^{33}$ Cf. Muñoz 2012. 
'esperanza'), siempre en el lugar más destacado, cómo incluso abandonó Sicilia por amor para casarse y morir en Roma, ${ }^{34}$ donde increpa a quienes se atrevan a profanar su tumba, nuevo lecho nupcial. Siendo una inscripción cristiana ya del siglo v, llama la atención la valiente expresión esperanza de pervivencia a través del amado, no de su Dios:

\begin{abstract}
Helpis dicta fui, Siculae regionis alumna, quam procul a patria coniugis egit amor, quo sine maesta dies nox anxia flebilis hora nec solum caro sed spiritus unus erat; lux mea non clausa est tali remanente marito maiorique animae parte superstes ero.

Porticibus sacris iam non peregrina quiesco, iudicis aeterni testificata thronum, ne qua manus bustum violet nisi forte iugalis. $[\ldots]$
\end{abstract}

(ICUR II, 4209 = CLE 1432, basílica de San Pedro Apóstol, vía Cornelia) ${ }^{35}$

Élpide es, como Medea o como una heroína de la novela griega, una mujer que por su amor y fidelidad es capaz de abandonar su tierra, la que, con todo, recuerda con nostalgia aún después de muerta en el primer verso. Recuerda a Platina, ante todo "una mujer de rara fidelidad y extraordinaria virtud" en la novella del relato de Hemo (App., Met., 7.6: "rarae fidei atque singularis pudicitiae femina"; 7.7 "unicae fidei"), esposa de un magistrado y madre de diez hijos que sufre avatares y aventuras por él, aguantando ingenio masculo hasta el punto de vestirse como un hombre y cortarse el pelo para rescatarle, intercediendo incluso

34 También en el breve epitafio de la virgo cristiana enterrada en Tarragona Tecla se indica su origen egipcio (el de la santa del mismo nombre): "Haec hic beata Thecla / virgo Chr(isti) ei patria Aegypt(us) / vixit ann(os) LXXVII ut meru/it in pace requievit d(omi)ni" (Hispania Epigraphica, 10, 2000, n 600).

35 "Élpide fui llamada, procedente de la región de Sicilia, a quien el amor de mi esposo guió lejos de mi patria, sin el cual el día me resulta triste, la noche angustiosa y la vida, en fin, digna de llanto; con él estaba unida en cuerpo y alma. Mi vida no ha concluido porque, permaneciendo vivo un marido semejante, también yo seguiré viviendo con la mayor parte de mi alma. En estos pórticos sagrados no descanso ya como extranjera y de ello da fe este trono del juez eterno, para que ninguna mano profane mi sepulcro, a no ser que mi esposo...". 
ante el César; del otro lado, el bandolero narrador, el mismo que pronuncia el elogio, también es obligado a disfrazarse de mujer para salvarse (cf. Ach. Tat., 6.1).

Mujeres tan valiosas merecen en justicia monumentos excepcionales. Uno de los mejores ejemplos de ello en Roma lo proporciona la conocida Laudatio Turiae, el más extenso elogio hasta ahora conservado, en dos enormes placas de mármol rotas en varios fragmentos (algunos de ellos perdidos), con suficiente espacio para al menos un retrato escultórico y una inscripción de más de 180 versos. ${ }^{36}$ En su descripción de las actividades públicas de quien conocemos como Turia - en realidad no se nombra en el texto conservado-, su esposo utiliza términos de acción y virtud propias de los hombres, aunque gran parte de la crítica se ha centrado en su entrega a éste y a su largo y armonioso matrimonio, ${ }^{37}$ gracias a su virtus, firmitas animi y constantia (valores propios en particular de héroes y gobernantes). ${ }^{38}$ Es el esposo quien la describe desde su perspectiva, con relación a él y los peligros de su propia e intensa vida pública: "speculatrix et propugnatrix meorum periculorum" (Laudatio Turiae, 2, 61); pero Turia es quien procura refuerzos (subsidia) como buena speculatrix y protectora (munibat) en su faceta de propugnatrix - atributo dedicado a Júpiter, con un templo en el Palatino (CIL VI, 2004, $2008,2009)-$, expresiones originales y a la vez próximas a aquellas metáforas militares que Ovidio emplea para animar a su esposa a la virtus (por ejemplo, en Pont., 3, 1, 91-94).

En suma, el superviviente se encarga de encomiar una fortaleza que puede animar a otras mujeres, apoyado en la buena reputación de la difunta y en el reconocimiento previo de la sociedad. ${ }^{39}$ El término fama ocupa precisamente el primer lugar absoluto en el elogio epigráfico que el papa Dámaso dedica a la virgen modélica por excelencia para los cris-

${ }^{36}$ Véanse la edición y estudio de referencia, con bibliografía anterior de Flach 1991.

${ }^{37}$ Así, Lefkowitz 1996, pp. 67-82, esp. pp. 77-79.

38 Sobre la virtus como concepto masculino aplicado de forma excepcional a las mujeres, cf. McDonnell 2006, pp. 161-165. En la poesía funeraria epigramática griega,

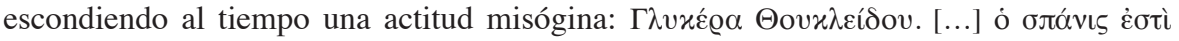

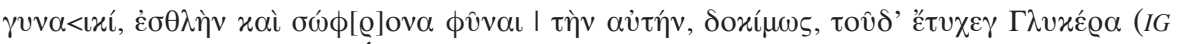
$\mathrm{II}^{2}, 11016={ }_{C E G} 525$, del Ática), "Glícera, hija de Tuclides. Lo que no es frecuente en una mujer, ser excelente a la vez que sensata, eso lo alcanzó Glicera" (trad. de Mª L. del Barrio).

${ }^{39}$ Cf. Cugusi 2013, pp. 233-249. 
tianos, Santa Inés; con esa función referencial que es un recurso propio de la poesía épica, reforzado por el adverbio dudum ('recientemente'), que confirma la firmeza del discurso que va a seguir:

Fama refert sanctos dudum retulisse parentes

(ICUR VIII, 20753, v. 1) $)^{40}$

Matronas y doncellas excepcionales sirven, apoyadas en su prudente firmeza, para afianzar un modelo de comportamiento convincente, atractivo y duradero para los miembros de su comunidad. Que ellas son ejemplo queda bien claro en el primer término de un epígrafe métrico del norte de África, para una difunta con otro nombre parlante:

Exemplum Matro/na sita est qua / fama ${ }^{41}$ loquetur sanctius et me/lius non potuisse / dari.

(CLE 2090, Blilia, Numidia $)^{42}$

De nuevo en su parte central se sitúa el discurso de la fama futura (fama loquetur), ${ }^{43}$ que contrasta con la mala fama de otras mujeres más conocidas, que llegó, por ejemplo, a hacer que Julio César se divorciara de su esposa, afectada por el escándalo de la fiesta de la Bona Dea (Plut., Caes., 10; Suet., Caes., 6.3).

Las inscripciones funerarias muestran que, entre la expresión del decus familiar más orgulloso y el exemplum más abierto a futuros lectores, la fuerza femenina resulta ser un elemento de admiración muy unido a otra cualidad discutida por los filósofos, su capacidad de hablar y comunicar sentimientos. Precisamente Aristóteles establece la diferente acepción de fortitudo de hombres y mujeres (así, en Pol., 1.13, 1260a22)

40 "La fama repite lo que ha poco declararon los santos progenitores de Inés".

${ }^{41}$ Ya en los epigramas funerarios griegos se destaca la buena fama que procede de la

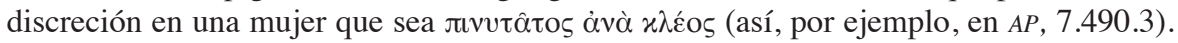
Más testimonios del uso de exemplum en inscripciones dedicadas a mujeres en Hernández 2001, pp. 158-159.

42 “Aquí está enterrada Matrona, un modelo de conducta, más respetable y mejor que la cual - tal es lo que se dice - no puede encontrarse".

${ }^{43} \mathrm{Cf}$. los siguientes paralelos, conocidos a través de la tradición manuscrita: "me fama loquetur / exemplum laudis vixi dum vita manebat" (CIL IX, 9, $4796=$ = CLE suppl. Riese, 437, vv. 15-16, de L. Merusio); "tuum nomen semper Karthago loquetur" (CLE 354 , v. 14, final del elogio en Cartago del venator Olimpio). 
$\mathrm{y}$, antes que San Pablo, considera que una de las virtudes que pueden desarrollar éstas de acuerdo con su naturaleza subordinada es el silencio. ${ }^{44}$ Es el silencio que pide Pericles en su "Oración fúnebre", cuando se dirige a las atenienses para explicarles que "será grande la reputación de aquella cuyas virtudes o defectos anden lo menos posible en boca de

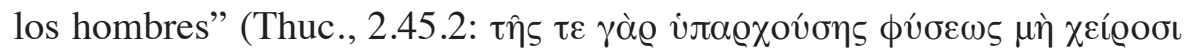

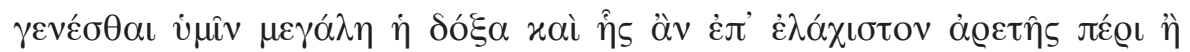

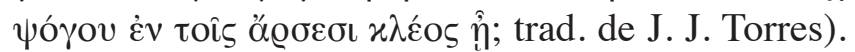

Tratados morales como el de Plutarco sobre los deberes del matrimonio también confirman que para la consecución de una relevancia ejemplar hay que restringir los discursos femeninos. Así, a propósito de una intervención pública de una mujer fuerte, Téano, esposa o hija de Pitágoras, es ella misma la autoridad que corta en seco un comentario motivado por el "imprudente" gesto de mostrar su brazo al ir a colocarse

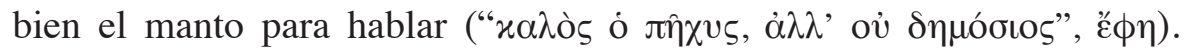
Esta anécdota da paso a un comentario que no sabemos si procede de la propia Téano o de Plutarco, que subraya que estas exposiciones, la física del propio cuerpo y la intelectual de la exposición de las palabras, no son apropiadas para la comunidad:

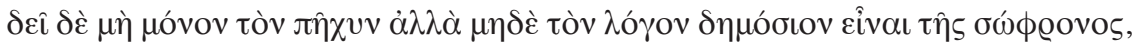

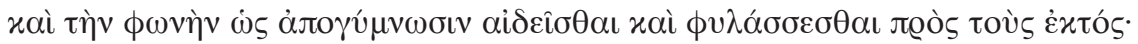

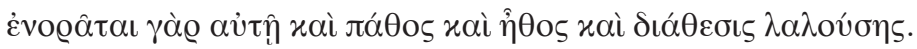

$(\text { Coniugalia Praecepta } 31)^{45}$

44 "Un modesto silencio hace honor a la mujer" expresa como un aforismo el verso 291 de Áyax de Sófocles, elegido para ilustrar el pasaje aristotélico sobre el poder doméstico (Arist., Pol., 1.5). Con estos fundamentos, Tomás de Aquino distinguirá el valor de los hombres, que sirve para gobernar (virtutes principiative), del de las mujeres, con un valor servil (virtutes ministrative). Cf. Bejczy 2011, pp. 266-268.

45 "Téano, colocándose el manto alrededor de su cuerpo, enseñaba el brazo. Cuando alguien le dijo "Hermoso brazo", ella le respondió "Pero no público". Conviene que no sólo el brazo, sino también el discurso de la mujer prudente no sean públicos; que ella sienta respeto y tenga cuidado de desnudar su palabra ante personas de fuera, ya que en la palabra se descubren los sentimientos, caracteres y disposiciones de la que habla" (trad. de Morales Otal y García López). La historia la refiere asimismo, Clemente de Alejandría, Strom., IV, 52 C, antes de una reflexión sobre las diferencias de los dos sexos motivadas por, entre otros factores, una diferente oportunidad a la hora de aprender. 
De hecho, en las Vidas Paralelas de Plutarco sólo leemos once intervenciones - sin excepción de la élite- de mujeres griegas y romanas. ${ }^{46}$ Sin acabar de romper esa proporción, las inscripciones latinas dejan asomar la voz propia de vez en cuando, bien sea de las propias difuntas, que repasan su vida o aconsejan, bien sea de las supervivientes, dirigiendo al viator/lector un discurso "epigráfico" que llega a reproducir un breve elogio funerario público. No entraremos en este momento en los ejemplos de mujeres ilustres que también esporádicamente aparecen entre los poetas, historiadores y biógrafos (así, Ov., Tr., 3.7; Plut., Pomp., 55), ya que desde los más antiguos testimonios en las inscripciones latinas las mujeres hablan como madres o hijas, pocas veces como partícipes de la vida pública, vinculadas a la religión:

Iunone Reg[ina] / matrona / Pisaurese / dono dedrot

(CIL I² 378, principios del siglo II a. C., en Pisaurum, Umbría)

Numis[iae] / Secunda[e] / Sabina[e] / Claudi Liber[alis] / sacerdoti Aug(ustae) m[atri] / municipii et colon[iae]

(Ann.Épigr., 1998, 416)

Por su consideración del silencio femenino incluso como signo de devoción (Aug., serm. 99: "tacita loquebatur; non sermonem promebat, sed deuotionem ostendebat"), no son demasiadas las mujeres con voz propia en la literatura cristiana, que intentan evitar en ellas un adulterium linguae (Hier., epist., 22.6). En efecto, desde los Padres de la Iglesia se divulga la consideración de que la formación femenina con vistas al ascetismo y el monacato ha de limitar las manifestaciones verbales y artísticas desde la infancia: "Nec tibi diserta multum velis videri aut lyricis festiva carminibus metro ludere" (22.9).

Con todo, y con el tono coloquial acentuado por las incorrecciones, la llana Julia reivindica su nombre con orgullo ${ }^{47}$ y su origen romano, para incluir en el último lugar su condición de neophyta, después de

${ }^{46}$ Cf. Buszard 2010.

${ }^{47}$ Frente a la actitud de Fauna o Hedua, predictora de los hados de las mujeres que "tenía tanto pudor que ningún mientras vivió hombre, salvo su marido, la vio ni oyó su nombre" (Inst., 1.22: "Varro scribit tantae pudicitiae fuisse, ut nemo eam, quoad uixerit, praeter uirum suum mas uiderit, nec nomen eius audierit"); sus seguidoras sí parece que en público la nombran y divinizan (idem: "illi mulieres in operto sacrificant, et bonam deam nominant"). 
haber conseguido, con el breve adverbio mox colocado antes de las expresiones formulares cristianas que suponen la aceptación de la muerte, resumir el tópico de la mors immatura:

Fuit mihi natibitas Romana nomen si queres

Iulia bocata so, que vixi munda cum byro meo

Florentio cui demisi tres filios superstetes

mox gratia dei percepi suscepta in pace neofyta.

$(I C U R \mathrm{IV}=$ CLE 1874)

Lejos está Julia de merecer los elogios de Venancio Fortunato de las manifestaciones públicas de Radegunda, que se refieren a sus capacidades musicales. Venancio evoca en un hexámetro la santidad melódica de la elocuencia de la reina en las celebraciones en honor a San Martín, que hacen de ella digna merecedora, por sus propios méritos, de ser largo tiempo recordada (diu ... diu; carmina ... carmina):

Carmina sancta diu uox illa ministret ab ore et recreent animos carmina secta diu.

(Ven. Fortun., Carm., app. 19.13-14, final del poema)

El celebrado poeta de corte y obispo de Poitiers está más cerca de lo que pretende el autor anónimo de un carmen epigraphicum, que pide una melodía para recordar a Sabina, de joven y bella voz silenciada por su muerte, encerrada su capacidad por su propia tumba (clausa iacet, v. 1):

vox ei grata fuit, pulsabat pollice cordas.

Set cito rapta silet. [...]

Et pia voce cane: Aelia Sabina vale.

(CLE 489, vv. 3-4 y 9, de Panonia $)^{48}$

El mejor homenaje es para Sabina una melodía que recuerde su nombre completo, cumpliendo así la función el epitafio (cane... vale). Más pretensiones tiene una puella fortis, que supuestamente saca con dificultad fuerzas de su flaqueza para elogiar al influyente y poderoso hermano

48 "Instruida en tantas habilidades, que fue la única que logró superar a su marido. Tuvo una hermosa voz y tocaba los instrumentos de cuerda con sus dedos. Pero, arrebatada rápidamente, guarda silencio”. 
difunto que merece un carmen. Éste opta por la tercera persona, pero el último término, dixit, confirma la voluntad como autora de Paula. Además, su falsa modestia es evidente, en cuanto que su despedida adopta el sencillo, emotivo y muy efectivo final del poema dedicado por Catulo también a su hermano, muerto en Troya (101.10). ${ }^{49}$ Ello completa nuestra idea de que se presenta como una Antígona romana, que cuida por el enterramiento digno de su hermano, otra vez sin ningún elemento propiamente cristiano y sí con la expresión del orgullo por los ancestros $\mathrm{y}$ por las acciones nobles que permiten el mantenimiento del vigor familiar. De hecho, es de nuevo el noble origen el motor que impulsa a la mujer a intentar abandonar ese carácter - se sobreentiende 'débil' femenino que hasta ese momento ha vencido en ella, como si la vida se tratara de una batalla (femineo victa animo ${ }^{50}$ ):

Consul in egregiis bis senis fascibus auctus, magnus ab Insteiis gens inclyta Pompeianus istic terrenos terrenis sedibus artus reddidit, inque sinus summi genitoris apertum aethera pervolitans levibus se sustulit alis caeloq(ue) et terris placida sic pace repostus felix luce nova saec(u)lorum in saecula gaudet femineo sed victa animo et miserabile dulci germano divulsa dolens fratremq(ue) requirens Paula soror tumulum dedit et solatia magni parva tulit luctus tristiq(ue) heu pectore 'salve perpetuumq(ue) vale, frater carissime' dixit.

$$
(C I L \mathrm{VI}, 32000(\mathrm{p} 4801)=\text { CLE } 734=I C U R \mathrm{I}, 307)^{51}
$$

${ }^{49}$ Muñoz 2011, pp. 60-62, reúne paralelos epigráficos, que son antecedente de numerosas evocaciones más en la literatura moderna de este verso, explicable por el poder de su simplicidad.

${ }^{50}$ Cf. Ov., Epist., 19.5. Val. Max., 4.6.1, impar femineo animo. Aún en La Celestina la doliente Melibea, antes de trasgredir la norma, se lamenta de esa fragilidad y, sobre todo, de la imposibilidad de las mujeres de expresar públicamente sus sentimientos: "O género femíneo, encogido y frágile! ¿Por qué no fue también a las hembras concedido poder descobrir su congoxoso y ardiente amor, como a los varones?” (10.426).

51 "Cónsul engrandecido con los doce haces consulares, el magno Pompeyano, ínclita descendencia de los Insteyos, devolvió aquí, a esta morada terrenal, su cuerpo también terrenal y volando a través del cielo hacia el seno acogedor del sumo Padre, se ha ido elevando con sus alas ligeras y así en el cielo como en la tierra disfrutando 
El discurso funerario se ve modificado por el dolor. Así, el apóstrofe de una madre ( $O$ Rhode) y las dos preguntas que le dirige a su hija difunta se rematan con la misma sencillez del epitafio de Catulo (iam vale perpetuo dulcis), convenientemente cristianizado con un añadido meramente formular, con un cierre perfecto en modo imperativo (in pace quiesce):

O Rhode, dulcis anima, acervo mihi funere rapta, qui tantum properasti matris foedare senectam senilemque aetatem tantos onerare dolores te sine namque mihi nec lux nec vita iocunda est, Quid primum tollerare queam tua dum singula quaero cum venit in mentem quod tuorum tu decus omnium esses?

Quid pudor castus quid sancta fides moresque benigni ingeniumque doctrinaque tua et verba sobria menti?

Prudens et innocua caelestia regna petisti Iam vale perpetuo dulcis et in pace quiesce!

$(\text { ICUR IX, } 24125=\text { CLE 737 })^{52}$

Después del epitafio en prosa con los datos biométricos (filiae... mater), otra madre afligida (genetrix decepta) toma la palabra y "reivindica" la preparación del titulus: ${ }^{53}$

ya de una paz inalterable, dichoso por haber alcanzado una vida nueva, se regocija por los siglos de los siglos. Pero su hermana Paula, vencido su ánimo femenino y separada a la fuerza de su pobre y querido hermano, llena de sufrimiento y sin dejar de echarlo de menos, le ha ofrecido esta tumba y le ha llevado un pequeño consuelo a tan gran dolor; y, ¡ay!, con todo el dolor de su corazón le ha dicho: «Salud y adiós para siempre, queridísimo hermano»").

52 “Oh, Rode, alma mía, arrebatada a mí por la prematura muerte, tú que tanto te apresuraste en arruinar la vejez de tu madre y colmar su edad senil de grandes sufrimientos. Pues sin ti no me resultan alegres ni la luz del día ni la propia vida. ¿En primer lugar, cómo voy a poder soportar mientras recuerdo cada una de tus cosas, cuando me viene a la mente el que tú serías el orgullo de todos los tuyos? ¿Y qué decir, sin desfallecer, de esa santa virtud tuya, esa sagrada fe y tu bondadosa conducta y tu talento y tu saber y tus palabras? Llena de prudencia y sin haber causado daño a nadie, te has dirigido hacia los reinos del cielo. Adiós ya para siempre, querida, y descansa en paz".

53 Véase el epitafio escrito con "las palabras de un padre": "verba patris / natum abere destinavi nono $\mathrm{m}$ [ense] / dedi animam dulcem tam benig[ne] / rapta est innocens quid maer[oris no]/ndum nulla poena expertus e[st] / fuerat non creare quam crea[re melius]" (Ann.Épigr., 1997, 410). 
Diis Manibus

Atiliae Severillae filiae piissimae

Atilia Onesime mater

hunc titulum natae genetrix decepta paravi

post $\mathrm{t}[\mathrm{en}] \mathrm{er}[\mathrm{os}]$ Manes et acerbae funera mortis

$[\ldots]$

$(C I L 11,01209=C L E 1550, \mathrm{vv} .1-2 \text {, época de Adriano })^{54}$

Aunque el final de la inscripción es muy fragmentario, la hija parece responder, pidiendo que se le permita a la madre que acaba de hablar, poder durante mucho tiempo realizar los ritos de la muerte y pronunciar su discurso, consciente de que así se mantendrá su vida terrenal a través de la memoria nominis: dum venio matris funere tarda comes (ibid., v. 20) ${ }^{55}$

El dolor es un motor asimismo para otra madre romana que escribe el monumento (memoria) a su hijo, muerto a los veinticinco años, añadiendo una amenaza o imprecatio, con pena justamente de "dolor" a quien pudiera atreverse a quitar la inscripción, su personal conmemoración, último vínculo afectivo entre ambos. Su nombre queda bien destacado en el campo epigráfico, reservándose la primera línea completa:
Claudia
Monim(a)e filio piissimo
memoriam scripsit
quicumque eum titu
lum velet removere
eum dolorem habeat.

A modo de diálogo intemporal, se llega a evocar en piedra una frase de la esposa, pronunciada cuando ya ha muerto (prior... recepta), que no es sino una personalización de imperativos como vivite, ludete, agite, etc., que amplían y divulgan el tópico del carpe diem, aquí muy próxima al último dístico de un epigrama de Marcial dirigido a Lucano (1.36.5-6: “... qui prior isset ad umbras / vive tuo, frater, tempore, vive meo"):

54 "Yo, su madre desolada, he preparado este epitafio para mi hija tras su muerte prematura y la cruel ruina que ha acabado con ella".

55 “.. hasta que yo, con el paso del tiempo, me convierta en compañera de mi madre a la hora de su muerte". 
prior at Lethen cum sit Pompti[11]a recepta tempore tu dixit vive Philippe $\mathrm{m}[\mathrm{e}] \mathrm{o} .{ }^{56}$

$(C I L \mathrm{X}, 7563$, etc. $=C L E 1552, \mathrm{c}, \mathrm{vv} .3-4$, Cagliari $)$

Otra de estas mujeres excepcionalmente conocidas gracias a las inscripciones que para ellas se grabaron incluso se atreve a asumir con insolencia su nueva situación. Así, los ecos de un epigrama griego atribuido a Epicarmo reviven en dos poemas epigráficos, en los que la difunta es mujer:

cinis sum, cinis terra est, terra dea est, ergo ego mortua non sum. ${ }^{57}$

$(C I L$ VI, $29609=$ CLE 974, s. I d. C. $)$

El último, un hexámetro asimismo de Roma (con los arcaizantes heic y sein, una contracción terrast y un final con dos monosílabos), recalca el orgullo de esa mujer sabia que se mantiene viva en la naturaleza que nutren sus cenizas:

Cara mieis vixi virgo vitam reddidi

Mortua heic ego sum et sum cinis, ${ }^{58}$ is cinis terrast, sein est terra dea, ego sum dea, mortua non sum..$^{59}$

$(C I L \mathrm{VI}, 35887=$ CLE 1532).

En fin, tanto las inscripciones latinas tanto métricas como las más humildes en prosa subrayan ocasionalmente, casi sin excepción en primera persona, valores considerados masculinos como la fortaleza, la justicia y la elocuencia, con una finalidad fundamentalmente consoladora que,

56 “....al ser acogida primero Pomptila en las aguas del Leteo dijo: «Vive tú, Filipo, en mi lugar»...".

57 "Soy ceniza, la tierra es ceniza; la tierra es una diosa, luego yo no soy una muerta" (trad. Rodríguez-Pantoja 2006, 889). Este silogismo se remonta, evidentemente, a un bastante más descarnado epigrama griego, atribuido a Epicarmo y conocido gracias a un

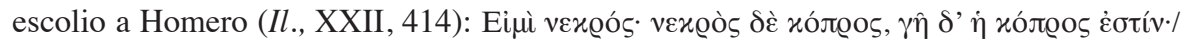

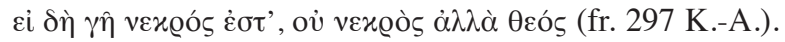

${ }^{58}$ Cf. Prop., 2.11.5-6: "et tua transibit contemnens ossa viator, / nec dicet 'Cinis hic docta puella fuit'".

59 "Muerta aquí estoy yo y soy ceniza; ceniza es la tierra; / pues si la tierra es diosa, yo soy diosa, no muerta" (trad. Rodríguez-Pantoja). 
sobre todo en las inscripciones cristianas, sirve además para completar un nuevo modelo de comportamiento.

Liberada la mujer de su natural molicie e irresolución, es posible una inversión en los papeles, según ya defiende sin desprecio Casiodoro, acabados ya el Imperio y un largo tiempo:

Nam et uiri cum mollescunt, animo femineo sunt; et mulieres uiriles efficiuntur, cum in bono proposito mentis robore perseuerant.

(Cassiod., Expositio psalmorum, 1)

\section{BIBLIOGRAFÍA}

\section{Autores y textos antiguos}

Anthologia latina sive poesis latinae supplementum. Pars posterior, Carmina epigraphica. Fasciculi I, 1.2; II, 1-2; III, Carmina latina epigraphica, ediderunt Franciscus Buecheler et Alexander Riese, conlegit Franciscus Buecheler, Lipsiae, in Aedibus B. G. Teubneri, 1895-1930. [CLE]

Anthologia Latina sive Poesis Latinae Supplementum. Pars prior: Carmina in codicibus scripta, recensuit Alexander Riese, fasciculus I; Libri Salmasiani aliorumque carmina, Lipsiae, in Aedibus B. G. Teubneri, 1869. [CLE Suppl. Riese]

Comoediarum togatarum fragmenta (in aliis scriptis seruata), vol. II: Comicorum fragmenta, Leipzig, Teubner [O. Ribbeck, 2da. ed. 1873].

Corpus inscriptionum Graecarum, edidit Augustus Boeckhius, Berolini, ex Officina Academica, 1828-1877. [IG]

Corpus inscriptionum Latinarum, Berolini, apud Georgium Reimerum, 1862- . $[\mathrm{CIL}]$

-, Poesía epigráfica latina I-II, introducción, traducción y notas de C. Fernández Martínez, Madrid, Gredos, 1988 (Biblioteca Clásica Gredos, 259-260).

Further Greek Epigrams: epigrams before A.D. 5o from Greek Anthology and other sources not included in "Hellenistic epigrams" or "The garland of Philip", edited by D. L. Page, revised and prepared for publication by R. D. Dawe and J. Diggle, Cambridge, Cambridge University Press, 1981. [AP]

-, Epigramas funerarios griegos, traducción, introducción y notas de $\mathrm{M}^{\mathrm{a}}$ Luisa del Barrio Vega, Madrid, Gredos, 1992 (Biblioteca Clásica Gredos, 163).

Hamdoune, Chr., et al. (eds.), Vie, mort et poésie dans l'Afrique romaine d'après un choix de Carmina Latina Epigraphica, Bruxelles, Latomus, 2011 (Collection Latomus, 130).

Inscriptiones christianae Italiae. Regio IX. Liguria reliqua trans et cis Appenninum, introduzione, edizione e commento a cura di G. Mennella-G. Coccoluto, Bari, Edipuglia, 1995. [ICI IX] 
Inscriptiones christianae urbis Romae septimo saeculo antiquiores, nova series I-X, colligere coepit Iohannes Baptista de Rossi, compleverunt et ediderunt Daniluus Mazzoleni et Carolus Carletti, Roma-in Civitate Vaticana, Pontificium Institutum Archaeologiae Christianae, 1922-1992. [ICUR]

Inscriptiones Latinae Christianae Veteres, edidit Ernestus Diehl, I-III, Berolini, Weidmanos, 1925-1967 [reimpr. Zurich, Weidmann, 1970]. [ILCV]

Passio Perpetuae et Felicitatis, ed. J. A. Robinson, The Passion of S. Perpetua, Cambridge, Cambridge University Press, 1891.

Petrus Cellensis, Commentaria in Ruth, ed. G. de Martel (CCCM 54), Turnhout, Brepols, 1983.

Plutarco, Vidas Paralelas. IV (Arístides-Catón; Filepemén-Flaminino; Pirro-Mario), introducción, traducción y notas de J. M. Guzmán Hermida y Ó. Martínez García, Madrid, Gredos, 2007 (Biblioteca Clásica Gredos, 356).

-, Obras morales y de costumbres (Moralia). II, Sobre la fortuna. Sobre la virtud y el vicio. Escrito de consolación a Apolonio. Consejos para conservar la salud. Deberes del matrimonio. Banquete de los siete sabios. Sobre la superstición, introducciones, traducciones y notas por C. Morales Otal y J. García López, Madrid, Gredos, 1986 (Biblioteca Clásica Gredos, 98).

TuCíDIDES, Historia de la guerra del Peloponeso, Libros I-II, introducción general de J. Calonge Ruiz, traducción y notas de J. J. Torres Esbarranch, Madrid, Gredos, 1990 (Biblioteca Clásica Gredos, 149).

Wagner, C. J., Polyglotte Tobit-Synopse. Griechisch, Lateinisch, Syrisch, Hebraïsch, Aramaïsch; mit einem Index zu den Tobit-Fragmenten vom Toten Meer, (Abhandlungen der Akademie der Wissenschaften in Göttingen; PhilologischHistorische Klasse, 3. Folge, Bd. 258; MSU XXVIII), Göttingen, Vandenhoeck und Ruprecht, 2003. [= TOBÍAs]

\section{Autores modernos}

BeJczy, I. P., The Cardinal Virtues in the Middle Ages: A Study in Moral Thought from the Fourth to the Fourteenth Century, Leiden, Brill, 2011.

Benzina-Ben AbDallah, Z.-L. Ladjimi-Sebaï, "Egregiae memoriae filia? À propos d'une inscription inédite d'Haïdra (Tunisie)", Antiquités africaines, 11, 1977, pp. 161-165.

Buszard, B., "The Speech of Greek and Roman Women in Plutarch's Lives", Class.Phil., 105, 1, 2010, pp. 83-115.

Совв, L. S., Dying to Be Men. Gender and Language in Early Christian Martyr Texts, Columbia, Columbia University Press, 2008.

Cugusi, P., “ ‘CLE 1988 (= CIL VI, 37965)', l'epigramma longum e l'elegia. Qualche osservazione metodologica sui testi epigrafici versificati”, Epigraphica, 75, 2013, pp. 233-249.

Flach, D., Die sogenannte Laudatio Turiae, Darmstadt, Wissenschaftliche Buchgesellschaft, 1991. 
Gascou, J., "Inscriptions de Tebéssa”, Mélanges d'Archéologie et d'Histoire, 81, 1969, pp. 537-599.

Gleason, M. W., Making Men: Sophists and Self-Representation in Ancient Rome, Princeton, Princeton University Press, 1995.

Hernández Pérez, R., Poesía latina sepulcral de la Hispania romana: estudio de los tópicos y sus formulaciones, València, Universitat de València, 2001.

LefKowitz, M. R., "Wives and Husbands", en I. McAuslan-P. Walcot (eds.), Women in Antiquity, Oxford, Oxford University Press, 1996.

McDonnell, M. A., Roman Manliness: Virtus and the Roman Republic, Cambridge, Cambridge University Press, 2006.

MuÑoz GARCíA DE ITURRosPe, M $^{\mathrm{a}}$ T., "Frater ave atque vale (Catull. 101.10): fortuna literaria de una fórmula epigráfica”, Studia Philologica Valentina, 13, n. s. 10, 2011, pp. 159-179.

- , "Mujeres religiosas en la Hispania Cristiana y visigoda: de la virgo a la univira”, en J. Martínez Gázquez et al. (coords.), Estudios de Latín Medieval Hispánico, Firenze, Sismel, 2012, pp. 95-107.

Prescendi, F., "Le deuil à Rome: mise en scène d'une émotion", Revue de l'histoire des religions, 225, 2008, pp. 227-313.

RodríGuez-PANTOJA, M., "Traducciones del griego al latín en la poesía epigráfica”, en E. Calderón et al. (eds.), Koinòs Lógos, Murcia, 2006, vol. 2, pp. 887-896.

Weitbrecht, J., "Maternity and Sainthood in the Medieval Perpetua Legend", en J. N. Bremmer y M. Formisano (eds.), Perpetua's Passions. Multidisciplinary Approaches to the Passio Perpetuae et Felicitatis, Oxford-New York, Oxford University Press, 2012, pp. 150-166.

Woolf, V., "On not Knowing Greek”, en The Common Reader: First Series, San Diego, Harcourt, Brace and Co., 1986 [1925]. 